\title{
Transverse Needling After Autologous Mini-Punch Grafts Improves Repigmentation in Stable Non-Segmental Vitiligo
}

\author{
Magdy Ragab' \\ Omneya El zagh ${ }^{2}$ \\ Carmen Farid (iD) \\ 'Department of Dermatology, \\ Venereology and Andrology, Faculty of \\ Medicine, Alexandria University, \\ Alexandria, Egypt; ${ }^{2}$ Department of \\ Dermatology, Venereology and \\ Andrology, Ministry of Health Hospitals, \\ Alexandria, Egypt
}

Background: Repigmentation remains the primary target in vitiligo treatment. Melanocyte transfer procedures are often required for repigmenting stable, resistant vitiligo lesions necessitating procedural optimization and comparative evaluation. In the current study, we aimed to assess the additive value of weekly transverse needling sessions after mini-punch grafting for repigmenting stable non-segmental vitiligo lesions versus either procedure alone. Methods: Eighty lesions, included in 20 stable non-segmental vitiligo patients, were randomly allocated to each of the three treatment groups (line-1, mini-punch grafting; line-2, needling; and line-3, combined grafting and needling) and to a fourth control group receiving non-procedural treatment (line-4). Oral mini-pulse steroids and narrow-band ultraviolet-B sessions were administered to all patients for 3 months before and 6 months after the interventions. The extent of repigmentation was assessed using planimetry. Secondary outcomes were the time to first repigmentation response, cosmetic matching, and patient satisfaction. Blinding and allocation concealment were not feasible owing to the intervention nature and within subject design.

Results: Mini-punch grafting followed by weekly needling for 6 months achieved the fastest response and highest extent of repigmentation. Mini-punch grafts and transverse needling alone provided better results than the control group. No steroid-associated side effects were reported. Conclusion: Weekly needling sessions after mini-punch grafting hastened and improved the repigmentation extent of stable, resistant, non-segmental vitiligo lesions and should be considered during treatment planning.

Keywords: vitiligo surgery, oral mini-pulse steroid, planimetry, narrow-band UVB, stability

\section{Plain Language Summary}

Loss of skin colour in vitiligo can be devastating, especially in darker coloured patients having exposed lesions. Restoring the colour in some vitiligo lesions is sometimes difficult using medical and ultraviolet therapies, necessitating resorting to surgery. In vitiligo surgeries, either pigmented skin grafts or viable pigment cells (melanocytes) are transferred from coloured to depigmented areas within the same patient through a variety of techniques. Understanding how different surgical techniques compare is important for informing clinical care. In the current work, we compared the repigmenting value of two procedural techniques for vitiligo, namely transferring small punched out grafts (mini-punch grafts (MPGs) and the needling (moving a fine needle horizontally between coloured and white areas), and a third approach which is combining both (needling sessions after MPGs) versus control lesions receiving no surgery. This was done in 80 vitiligo lesions contributed by 20 patients having stable disease and no contraindication to any of the procedures done. Patients additionally received oral low-dose steroid twice weekly, and narrow band ultraviolet B (NB-UVB) sessions all through the study. The combined MPGs/needling treatment gave the best percent 
repigmentation as calculated by an image analysis software, at a significantly shorter time, giving better patient satisfaction, and achieving better colour matching. Side effects' frequency was comparable to other lines except for a slightly higher incidence of disease activity. Hence, we recommend adding needling sessions after MPGs to improve and hasten the response. The outcome assessment was not blinded due to the intervention nature.

\section{Introduction}

Repigmentation remains the primary target in treating vitiligo. The absence of functioning melanocytes in the vitiliginous epidermis necessitates relying on local marginal and follicular melanocytes/melanoblasts for melanization. Hairless or white hair areas, which are deficient in follicular reservoir, would require a melanocyte transfer procedure (melanocyte-rich tissue grafting or cell suspensions) from distant uninvolved locations. ${ }^{1}$ Existent or transferred melanocytes can be further stimulated by narrow band ultraviolet-B (NB-UVB) light, ${ }^{2}$ platelet-rich plasma, ${ }^{3}$ mesenchymal stem cells, ${ }^{4}$ fractional CO2 laser, ${ }^{5}$ or microneedling ${ }^{6}$ with variable success. Well-designed studies comparing the effectiveness of different vitiligo surgeries are necessary to inform treatment and optimize outcomes. Our aim in this study was to assess the efficacy and tolerability of transverse needling sessions following mini-punch grafts versus either procedure alone, on the repigmentation of stable, non-segmental, vitiligo lesions.

\section{Materials and Methods Study Design and Ethics}

This was a comparative prospective study. Patients were recruited through the outpatient dermatology clinic at Alexandria Main University Hospital, Egypt, between February and December 2019. Informed written consent was obtained from all patients. The study was performed in accordance with the standards laid down in Declaration of Helsinki and all its amendments. It was approved by Alexandria Faculty of Medicine Ethical Committee (serial approval number: 0105586, IRB code: 00012098, FWA:00018699) and was registered at ClinicalTrials.gov (NCT03872804).

\section{Patient Selection}

The study group consisted of adult patients with stable, resistant, non-segmental vitiligo. Patients having segmental or universal vitiligo, associated autoimmune diseases, bleeding tendency, skin neoplasia, photosensitivity, hypertrophic or keloid scarring tendency, psychological instability, contraindications to corticosteroid therapy, and pregnant or breastfeeding women were excluded. Patients' clinical characteristics were all recorded focusing on disease and stability duration, clinical type, Vitiligo Area Scoring Index (VASI), Vitiligo disease activity (VIDA) score, and patients' skin phototype. Stability was defined over the prior 6 months as lack of; development of new lesions, enlargement of existent lesions, Koebner's phenomenon, trichrome or confetti lesions, and lesions with ill-defined borders. ${ }^{7}$ Resistance to therapy was judged in the initially recruited 35 patients after administering NBUVB light and low-dose oral mini pulse (OMP) dexamethasone ( $2.5 \mathrm{mg}$ on two consecutive weekly days) for 3 months. ${ }^{8}$ NB-UVB light treatment was started at a dose of $0.15-0.40 \mathrm{~J} / \mathrm{cm} 2$, according to the skin photo-type, with a $10-15 \%$ increment per session, as tolerated. ${ }^{9}$ Absent $(0 \%)$ or poor $(<25 \%)$ repigmentation after this regiment was defined as resistant vitiligo ${ }^{10}$; patients with greater repigmentation extent or showing signs of activity were excluded while some were missed during the follow up $(n=15)$, leaving only 20 patients to complete the study. The minimum calculated sample size to give an $80 \%$ power to the primary outcome of repigmentation extent was 14 per group.

\section{Treatment Assignment and Procedures}

Four comparable lesions per patient (regarding surface area and distribution) were selected providing 80 lesions for random assignment to one of our four treatment groups. Treatment line-1 consisted of 1-mm autologous mini-punch grafts (MPGs) transferred from the uninvolved pigmented skin of the gluteal area to the recipient lesion under topical anesthesia. Briefly, the punches were first performed in the recipient, vitiliginous area to the level of mid dermis (approximately $1-1.5 \mathrm{~mm}$ in depth) using $1 \mathrm{~mm}$ disposable punch graft instrument. The depigmented skin within these holes was pulled out with a forceps, cut with scissors and discarded. They were placed 5-8 $\mathrm{mm}$ apart and started right next to the lesional border. The recipient area was then covered with saline soaked gauze using pressure for hemostasis. In the donor gluteal area, punch impressions were made $1 \mathrm{~mm}$ apart to maximize the number of grafts taken. The minigrafts were placed in normal saline, till getting inserted into the recipient area chambers with the dermal portion of the graft in direct contact with the dermis of the recipient perforations. They were pressed firmly in place using saline-soaked gauze, and secured using Steri- 
$\operatorname{strip}^{\circledR}(3 \mathrm{M}$, St Paul, MN, USA) and a three-layer dressing that from inside out included paraffin-embedded sterile gauze, sterile Pharmapore ${ }^{\circledR}$, and adhesive plaster for 7-14 days. ${ }^{2}$

Line- 2 consisted of transverse needling performed once weekly for 6 months (or until complete repigmentation). After application of a topical anesthetic, a 30-gauge disposable needle, mounted on an insulin syringe, was obliquely inserted close to the dermo-epidermal junction. Horizontal movements were then performed from multiple sites of insertion around the pigmented margins of the lesion towards the depigmented area and from any pigmented island within the lesion. ${ }^{11}$ Line-3 consisted of MPGs, performed as in line-1, followed by transverse needling sessions as in treatment line- 2 after leaving a 14-30 days interval for graft taking. Needling started from the lesional margins and from the MPG-colored islands within the lesion. No procedures were performed for the control lesions. Low-dose OMP and twice weekly NB-UVB light sessions for all lesions continued for a follow-up period of 6 months, giving a whole study duration of 9 months.

\section{Measured Outcomes}

The primary outcome was the extent of repigmentation quantified by planimetry (ImageJ, US National Institutes of Health, Bethesda, Maryland, USA). The surface area of the lesions was measured at baseline and at 3 and 6 months after treatment initiation, and repigmentation extent was reported as the percent change from baseline.

The secondary outcomes were as follows: time to first appearance of repigmentation (AOR); cosmetic matching to the surrounding skin, subjectively assessed by an examiner blinded to treatment allocation of the lesions and described as "excellent", "good", and "fair"; patient satisfaction, assessed using a 10-point visual analogue scale (VAS), with anchors at "0" (not satisfied at all) and "10" (completely satisfied); and type and frequency of complications.

\section{Statistical Analysis}

The normality of data was evaluated using the Kolmogorov-Smirnov test. Continuous variables were described using the mean (standard deviation, SD) or median (interquartile range, IRQ) as appropriate for the data distribution. Categorical variables were described as the count (percentage, \%). The anatomical distribution of lesion as well as the colour matching were compared between the groups using a chi-squared test with Monte Carlo correction. Marginal test of homogeneity was used to compare difference in categorical variables $(>2)$ between each pair of treatment applied to same patients. For quantitative variables measured repetitively along the follow-up, comparisons utilized Repeated measures ANOVA as well as Friedman's two-way analysis of variance. Adjusted $\mathrm{p}$ value by Bonferroni test was used for pairwise comparison of significant results. A mixed-design analysis of variance model was used to test for differences between two or more independent groups while subjecting participants to repeated measures. A two-tailed p-value $<0.05$ was considered significant. A power analysis was conducted to determine if the findings were clinically relevant. All analyses were performed using IBM SPSS Statistics (version 21).

\section{Results}

Out of the initially recruited 35 patients, 9 patients had activity, and 6 were lost to follow-up due to logistic reasons during the first 3 months, leaving only 20 patients to complete the intervention component of our study. Participants included 15 women (75\%), 70\% of the patients had generalized vitiligo, mean stability duration at inclusion was 19.2 \pm 15.3 months (Supplemental Table 1). The anatomical lesional distribution was not different between the three treatment groups and the control. Acral lesions constituted $65-75 \%$ of lesions while facial lesions were absent (Supplemental Table 2). The mean number of NB-UVB sessions over the 9-month period of the study was 70.9 \pm 1.1 , with a mean cumulative dose of $152.57 \pm 20.79 \mathrm{~J} / \mathrm{cm}^{2}$.

\section{Comparative Efficacy of the Treatment Lines}

Outcomes for the four treatment groups are summarized in Table 1. The AOR was significantly different between the four groups ( $p<0.001$ ), with the quickest lesions to repigment being those of combined needling and mini-punch grafts (line-3), followed by lines-1 (MPGs) and -2 (needling) respectively. The extent of repigmentation at 6 months was also highest for line-3 (median \% repigmentation, $49.6 \%$ ), followed by line-1 (38.5\%) and $-2(33.4 \%)$; the extent of repigmentation was lowest for lesions in the control group (5.5\%). An above $50 \%$ repigmentation was achieved in $45 \%$ of lesions in the line- 3 group, compared to $25 \%$ in line- $1,15 \%$ in line-2, and $10 \%$ in the control group. Results of planimetry over the 6-month follow-up are shown in Figure 1. 
Table I AOR and Extent of Repigmentation Among Treatment Groups

\begin{tabular}{|c|c|c|c|c|c|c|}
\hline Variable & Line-I & Line-2 & Line-3 & Line-4 & P-value & Power \\
\hline \multicolumn{5}{|l|}{ AOR (days) } & \multirow{2}{*}{$\begin{array}{l}\mathrm{F}_{\mathrm{r}}=16.6 \\
\mathrm{P}<0.001 *\end{array}$} & \multirow[t]{2}{*}{$80.8 \%$} \\
\hline Mean (SD) & $31.1(10.3)$ & $48.1(20.5)$ & $28.9(9.3)$ & $60.3(21.9)$ & & \\
\hline$P$ values & \multicolumn{6}{|c|}{$P I=0.003 * * P 2=0.00 I * * P 3=0.01 * *$} \\
\hline \multicolumn{5}{|c|}{ Percent pigmentation (0-6 months) } & \multirow{2}{*}{$\begin{array}{l}\mathrm{F}_{\mathrm{r}}=30.8 \\
\mathrm{P}<0.00 I^{*}\end{array}$} & \multirow[t]{2}{*}{$99.6 \%$} \\
\hline Median (range) & $38.5(I-85)$ & $33.4(0-77)$ & $49.6(4-97.2)$ & $5.5(0-70)$ & & \\
\hline$P$ values & \multicolumn{6}{|c|}{$P I=0.009 * * P 2=0.21 * * P 3<0.00 I * *$} \\
\hline
\end{tabular}

Notes: *Results $\leq 0.05$ are significant by related samples Friedman's two-way analysis of variance by ranks. **Results $\leq 0.05$ are significant in adjusted pairwise comparisons by Bonferroni test. PI, P2, P3 = significance of difference between lines I, 2, 3 respectively and control. Other pairwise comparison between groups are not statistically significant ( $>>0.05$ ). Abbreviations: SD, standard deviation; AOR, appearance of repigmentation.

The VIDA score significantly influenced the repigmentation extent among all four groups (F-interaction $=0.563$, $\mathrm{p}=0.034$ ), with a significantly lower extent of repigmentation among patients with a VIDA+1 score compared to those with a VIDA-0 score (Supplemental Table 3). By contrast, the effect of VASI score on the outcomes was not significant, despite the higher mean extent of repigmentation among patients with a VASI score $\leq 10 \%$ than among those with VASI score $>10 \% \quad$ (F-interaction=2.54, $\mathrm{P}=0.067$, Supplemental Table 3).

Patient satisfaction was significantly different between the 4 groups $(p<0.001)$, with the highest satisfaction achieved with line-3 (median VAS, 6.5/10), followed by line-1 (5.5/10) and line-2 (4.5/10). Patient satisfaction with control lesions was the lowest (2/10; Supplemental Table 4). Cosmetic matching to the surrounding skin did not differ significantly among the four treatment lines $(\mathrm{p}=0.38)$. The frequency of achieving "excellent" matching was highest for line-3, followed by line-1 and-2 (Table 2). Representative sequential changes in pigmentation over the follow-up period for each treatment group are shown in Figures 2-4.

Complications encountered included lesional activity occurring most frequently in line- 2 and manifesting as widening of the lesion $(n=3)$ or loss of marginal definition and pigmentation ( $\mathrm{n}=1$; Supplementary Figure 1). For line-3, there were two cases of partial and total graft and perigraft depigmentation (Figure 5). A marginal confetti lesion developed in one lesion in the control group. All patients

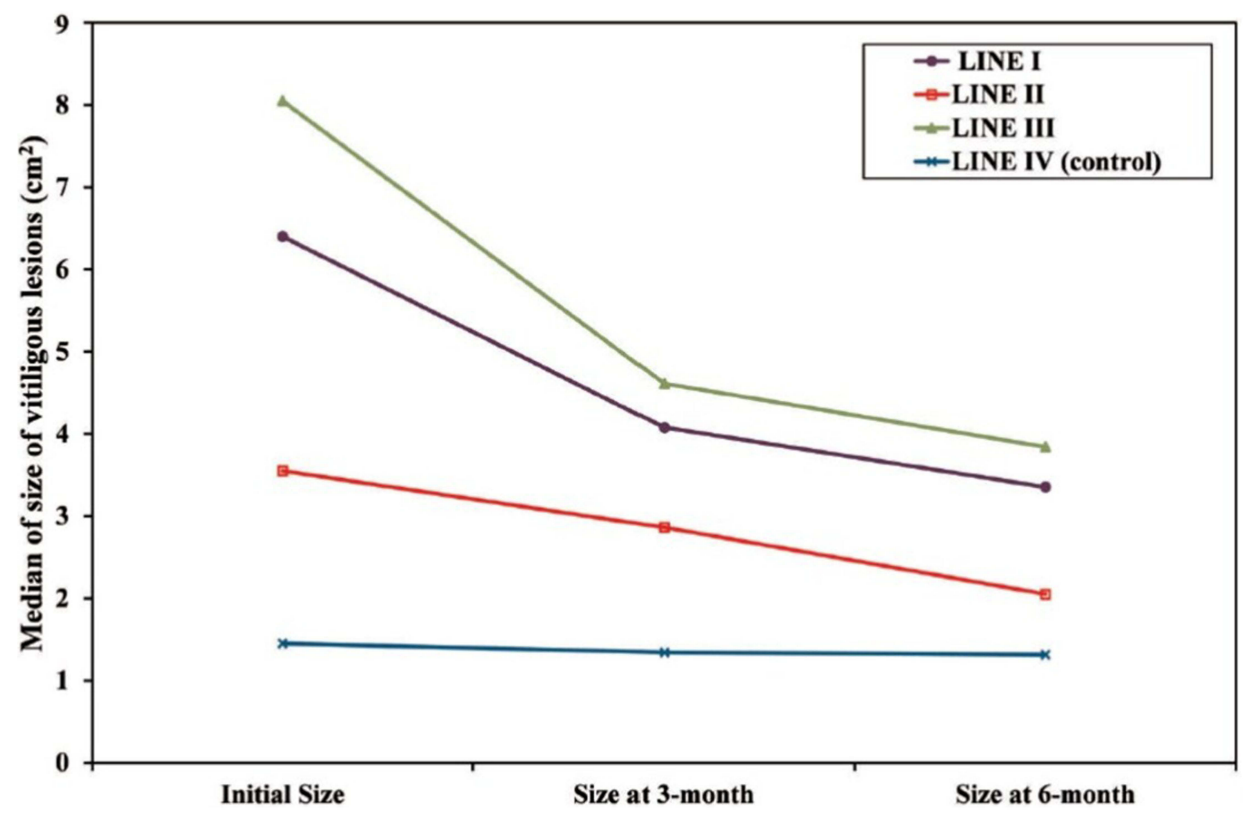

Figure I Changes in the lesional median surface area from baseline to 3 and 6 -months, for the treatment groups. 
Table 2 Cosmetic Matching Among the Four Lines of Treatment

\begin{tabular}{|l|l|l|l|l|l|l|l|l|}
\hline \multirow{2}{*}{$\begin{array}{l}\text { Cosmetic } \\
\text { Matching }\end{array}$} & \multicolumn{2}{|l|}{ Line-I } & \multicolumn{2}{|c|}{ Line-2 } & \multicolumn{2}{l|}{ Line-3 } & \multicolumn{2}{l|}{ Line-4 } \\
\cline { 2 - 9 } & n & $\%$ & n & $\%$ & n & $\%$ & n & $\%$ \\
\hline Fair & 3 & 15.8 & 2 & 13.4 & 2 & 11.1 & 4 & 36.4 \\
Good & 7 & 36.8 & 8 & 53.3 & 6 & 33.3 & 5 & 45.4 \\
Excellent & 9 & 47.4 & 5 & 33.3 & 10 & 55.6 & 2 & 18.2 \\
\hline P value & \multicolumn{6}{|c|}{$\#$ \#C $\mathbf{0 . 3 8 0}$} \\
\hline
\end{tabular}

Note: ${ }^{\#}$ Monte Carlo level of significance.

developing activity had an initial VIDA score of +1 . Partial graft loss occurred in four lesions in line-1 and one lesion in line-3, all of which were due to patient non-compliance with immobilization instructions. For line-1, cobble stoning developed in one lesion, which had improved by the 6-month endpoint of follow-up. Static grafts were seen in only one lesion in the line-1 group, yet follicular and marginal repigmentation occurred on subsequent phototherapy. Marginal hyperpigmentation occurred in $10 \%$ of the lesions in line-3 and 5\% in line-2; donor site hyperpigmentation occurred in three cases. Pain, ecchymosis, phototherapy skin burns, and contact dermatitis due to adhesive dressings occurred infrequently and were temporary, resolving without intervention. No steroid-related complications were observed during the study period. The raw data supporting these results (Master table) are indefinitely available at: https://figshare.com/ under the DOI https://doi.org/10.4121/ 14680980 .

\section{Discussion}

In the current study, we evaluated and compared multiple outcomes of two simple inexpensive procedures for vitiligo lesion repigmentation (MPGs and transverse needling), performed individually (lines- 1 and -2 , respectively) or in combination (line-3), in contrast to non-procedural treatment using a within-subject design to control for the confounding effects of age, disease duration and activity, skin phototype, and genetic background. ${ }^{8,12}$ The comparison aimed to

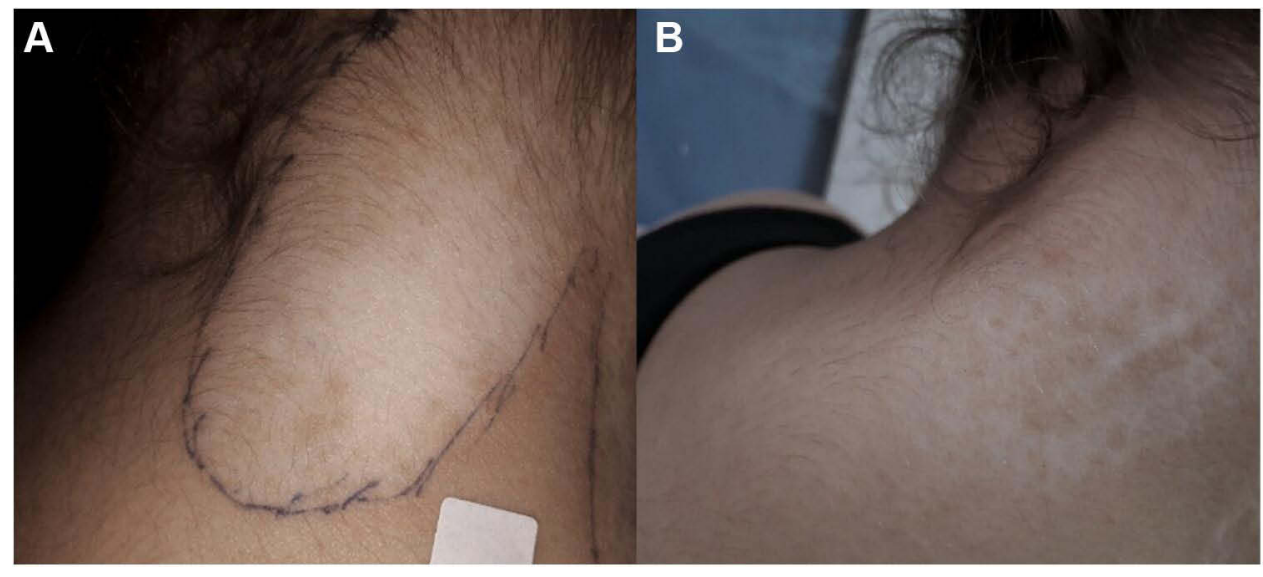

Figure 2 Vitiligo vulgaris lesion on nape of the neck treated by mini-punch grafts. (A) Baseline surface area of $7.3 \mathrm{~cm}^{2} .(\mathbf{B}): 47.9 \%$ Repigmentation after 6 months.

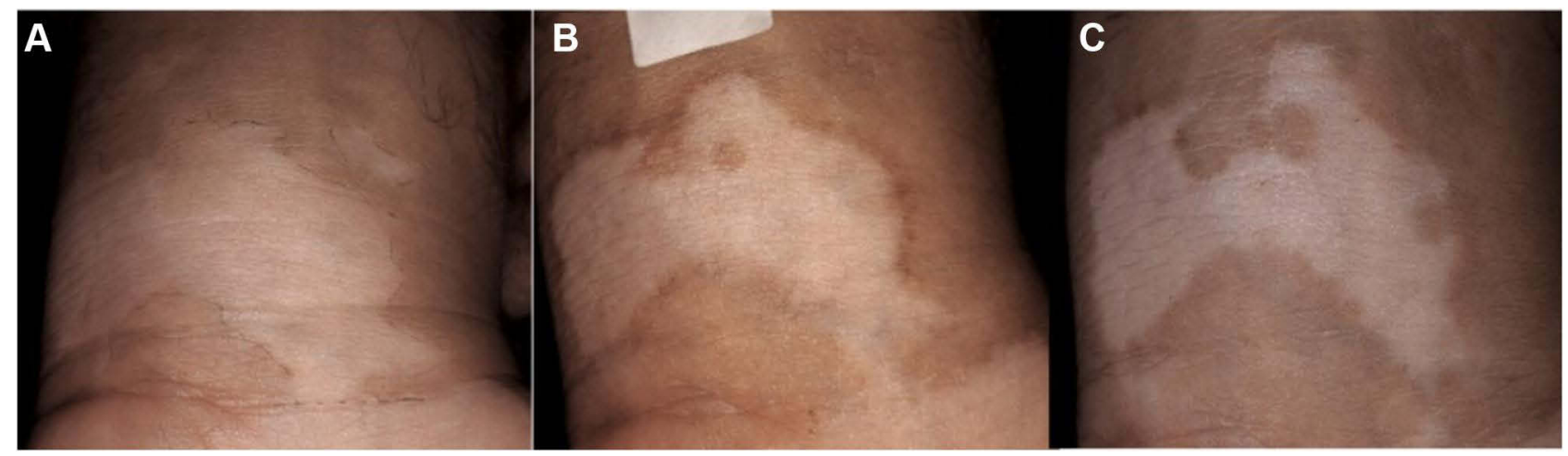

Figure 3 Vitiliginous lesion on left wrist treated with needling (A) initial surface area of $10.2 \mathrm{~cm}^{2}$. (B) $22.5 \%$ Repigmentation after 3 months, with partial marginal hyperpigmentation. (C) $51 \%$ Repigmentation at 6 months. 


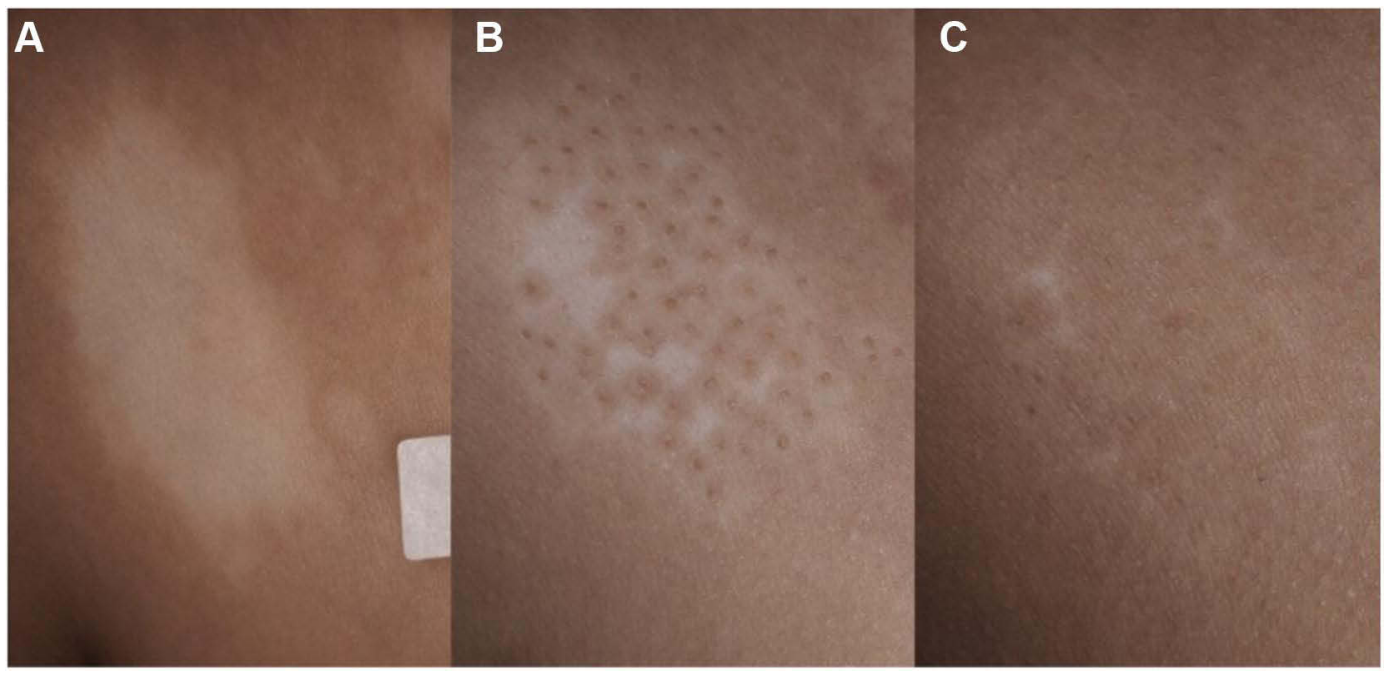

Figure 4 Trunkal vitiligo lesion treated with mini-punch grafts followed by transverse needling sessions. (A) Baseline surface area of $7.9 \mathrm{~cm}^{2}$. (B) $88.6 \%$ Repigmentation after 3 months. (C) $91.1 \%$ Repigmentation after 6 months with excellent color match.

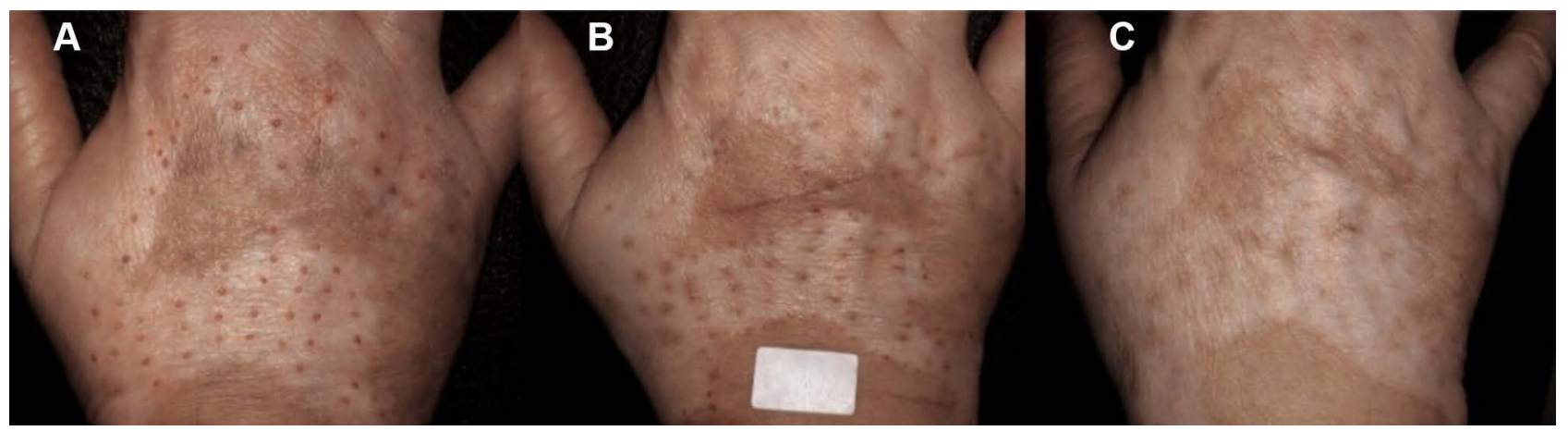

Figure 5 Loss of graft pigment due to lesional activity. (A) Baseline right-hand dorsum vitiligo lesion. (B) Perigraft pigmentation with good marginal definition at 12 weeks after mini-punch grafts followed by needling sessions. (C) Loss of pigmentation at and around the implanted grafts at 18 weeks indicating activity.

evaluate the efficacy and tolerability of these lines in repigmenting resistant, stable vitiligo lesions so as to aid in the choice of appropriate procedure. The sample size utilized gave a high power for most of the primary outcomes studied validating the conclusions. Low-dose OMP steroid therapy was used owing to its anti-inflammatory action to maintain disease stability against a possible Koebner's reaction to repeated procedural trauma and for aiding repigmentation. $^{8,12-15}$ Concerns about possible steroidassociated side effects as impaired wound healing and increased risk of infection ${ }^{16}$ might explain its scarce use alongside vitiligo surgeries; however, there were no cutaneous or systemic steroid-associated complications observed in our study, as similarly reported by previous researchers. $^{13,14}$

The majority of lesions treated in our study (65-75\%) were acral lesions since they are mostly resistant to treatment and represent the bulk of lesions indicated for surgery in actual practice, ${ }^{1}$ while the highly responsive facial lesions ${ }^{17}$ were not represented at all. This likely explains the somewhat lower repigmentation extents in our study compared to previous studies, which generally had a low representation of acral lesions, for example $6.6 \%{ }^{18}$ and $20 \%{ }^{19}$

The primary outcome of our study was percent repigmentation assessed by planimetry, which has been validated for use in vitiligo and alopecia areata. ${ }^{20}$ This method despite being accurate and reproducible, is technically demanding and time consuming, reducing its practicality for clinical practice and research, which might explain the scarce reports on this outcome to date. ${ }^{21}$ Scarcity in planimetry reports for assessing repigmentation necessitated the adoption of multiple other endpoints pertaining to speed of response, percentage of patients showing moderate to excellent $(50-100 \%)$ repigmentation, cosmetic 
matching, patient satisfaction, and technique tolerability, taking into consideration both physician and patients' perspectives. In general, a great heterogeneity was found in literature reporting the treatment outcomes in vitiligo, causing difficulty in comparing results. ${ }^{22}$

The clearly significant superiority of line-3, over lines1 and -2 and the no-procedure control, identified by planimetry (percent repigmentation), was confirmed by the secondary outcomes. Namely, line-3 provided the fastest time to response and greatest extent of repigmentation, at the lowest cumulative dose of NB-UVB, and providing the best cosmetic matching, with the highest patient satisfaction; all justifications to shift our practice to adopt needling sessions after MPGs in treated cases.

The greater extent of repigmentation might also allow the use of a smaller number of MPGs or increase their inbetween distance to cover a larger surface area, thus increasing the donor-to-recipient ratio. The quicker response also spares the patient NB-UVB sessions, providing a financial benefit and lowering the risk of associated side effects.

Further research is additionally needed to determine the value of needling following other types of vitiligo surgery, and the optimal frequency and duration of needling sessions, to achieve the best results. Of note, needling was initially used to enhance the delivery of topical medications for the treatment of vitiligo. ${ }^{6}$ Subsequently, both transverse and vertical needling were used to stimulate keratinocyte and melanocyte/melanoblast migration and proliferation in vitiligo lesions at the site of wounding. ${ }^{23}$ The mechanism of action of needling is two-fold: the associated inflammatory response, which releases stimulatory cytokines and growth factors, such as platelet-derived growth factor, transforming growth factor alpha and beta, and fibroblast growth factor, enhancing melanization, and a possible mechanical effect of pushing the melanocytes from pigmented to depigmented areas during transverse needling. ${ }^{23}$

To our knowledge, our study is the first to have evaluated the effect of combined MPG/needling; hence, comparison to literature is regrettably unfeasible. The main disadvantage of this technique was the higher frequency $(10 \%)$ of lesional activity, compared to line-1 $(0 \%)$ and the control group (5\%). This frequency, however, was lower than the $20 \%$ frequency for needling alone (line-2). Tissue resident memory cells (TRM) within lesional $\operatorname{skin}^{24}$ can be stimulated by the repeated trauma and inflammation consequent to needling causing activity as in lines- 2 and 3 . In line-3, grafting replaced areas of involved infiltrated lesional skin by normal skin, diluting the number of TRMs, explaining its lower frequency of lesional activity despite the needling trauma. Partial graft loss occurred in $5 \%$ on line- 3 lesions, while it was encountered in $20 \%$ of line-1 lesions. Both frequencies are comparable with literature reports made by Rajaram et $\mathrm{al}^{25}$ and Das et $\mathrm{al}^{26}$ reporting $22 \%$ and $10 \%$ frequency of graft loss, respectively. No cobblestoning occurred in line-3 cases, in contrast to a single case (5\%) in line- 1 . The use of $1-\mathrm{mm}$ punches at both donor and recipient areas, and the proper thickness of the graft due to trimming might have reduced this phenomenon, which is considered the most common complication with MPG amounting to around $40 \%$ in some studies. ${ }^{27}$

Marginal hyperpigmentation was more frequent in line-3 $(10 \%)$ due to the presence of both marginal active pre-existing and transferred melanocytes which were stimulated by needling, unlike the situation in lines-2 (5\%) and -1 (none). Cosmetic matching was excellent in more than $55 \%$ of lesion in the line-3 group, which was greater than for all other groups perhaps due to the higher extent of repigmentation and lower frequency of side effects encountered.

A significant disadvantage for this procedure despite its results is the frequent visits entailed for the weekly needling, which is both time and resource consuming for both the patient and the medical facility. Studies to evaluate lower frequency of sessions are needed, and considering the training of paramedical personnel on the technique could be resource saving.

MPGs achieved a repigmentation extent of $38.5 \%$, with $25 \%$ of lesions achieving above $50 \%$ repigmentation. No studies were found in literature depicting the extent of pigmentation after MPG using planimetry, most of the evaluation methods relied on subjective methods as physician global assessment (PGA) 5-point system, reporting the percent of patients achieving moderate to excellent pigmentation (50-100\%). For MPG, this number ranged between $40 \%$ and $85 \%{ }^{27,28}$ In most of those studies, the regional classification of the results was not included.

The extent of repigmentation was $33 \%$ for needling (line-2), with $15 \%$ of the lesions achieving the above $50 \%$ repigmentation threshold which is comparable to a reported rate of $17 \%{ }^{29}$ despite the acral majority of our lesions. In needling, melanocyte population of depigmented areas was not augmented by transferred ones, it only relied on activating pre-existent and marginal cells, 
explaining its relatively inferior results. Still, it had a significant advantage over non-procedural treatment employed in the control lesions.

For all lines, better responses were associated with a longer stability of lesions, which has been previously reported. ${ }^{30}$ Lesional stability and a lower VASI score might reflect a less severe immunopathology, allowing for a better treatment response. ${ }^{8}$

The limitations of our study include the small sample size; nevertheless, the power of all the main outcomes exceeded $80 \%$ indicating valid results. Not all vitiligo clinical types and distribution patterns were included. Double blinding and allocation concealment were not feasible for the current study design.

\section{Conclusion}

In summary, needling after MPG is superior to either grafting or needling alone for repigmentation of stable non-segmental vitiligo and is well tolerated. Future controlled blinded studies are needed to verify this conclusion and to determine the optimal frequency of needling, the value of using OMP steroids alongside surgery, and the value of needling following other types of melanocyte transfer.

\section{Acknowledgments}

Authors funded the work, and Alexandria Faculty of Medicine provided the workplace and facilities.

\section{Disclosure}

The authors report no conflicts of interest in this work.

\section{References}

1. Razmi TM, Afra TP, Parsad D. Vitiligo surgery: a journey from tissues via cells to the stems! Exp Dermatol. 2019;28(6):690-694. doi:10.1111/exd.13807

2. Mulekar SV, Isedeh P. Surgical interventions for vitiligo: an evidence-based review. Br J Dermatol. 2013;169(Suppl.3):57-66. doi:10.1111/bjd.12532

3. Chen J, Wan Y, Lin Y, Jiang H. Current art of combination therapy with autologous platelet-rich plasma for stable vitiligo: a meta-analysis. Int Wound J. 2020;27.

4. Esquivel D, Mishra R, Srivastava A. Stem cell therapy offers a possible safe and promising alternative approach for treating vitiligo: a Review. Curr Pharm Des. 2020;26(37):4815-4821. doi:10.2174/ 1381612826666200730221446

5. Kim WI, Kim S, Lee SH, Cho MK. The efficacy of fractional carbon dioxide laser combined with narrow-band ultraviolet-B phototherapy for non-segmental vitiligo: a systematic review and meta-analysis. Lasers Med Sci. 2021;36(1):165-173. doi:10.1007/s10103-02003069-0
6. Salloum A, Bazzi N, Maalouf D, Habre M. Microneedling in vitiligo: a systematic review. Dermatol Ther. 2020;33(6):e14297. doi:10.1111/ dth. 14297

7. Benzekri L, Gauthier Y. Clinical markers of vitiligo activity. $J \mathrm{Am}$ Acad Dermatol. 2017;76(5):856-862. doi:10.1016/j.jaad.2016.12.040

8. Kanwar AJ, Mahajan R, Parsad D. Low-dose oral mini-pulse dexamethasone therapy in progressive unstable vitiligo. $J$ Cutan Med Surg. 2013;17(4):259-268. doi:10.2310/7750.2013.12053

9. Mohammad TF, Hamzavi IH, Lim HW. Phototherapy and PUVA. In: Lim H, Kohen L, Schneider S, Yeager D, editors. Practical Guide to Dermatology. Cham: Springer; 2020:167-175.

10. Eleftheriadou V, Hamzavi I, Pandya AG, et al. International Initiative for Outcomes (INFO) for vitiligo: workshops with patients with vitiligo on repigmentation. Br J Dermatol. 2019;180(3):574-579. doi:10.1111/bjd.17013

11. Ahmad T, Rashid T, Rani Z. Needling: an adjunct to narrowband ultraviolet-B therapy in localized fixed vitiligo. J Pak Assoc Dermatol. 2016;18:149-153.

12. Lahiri K, Malakar S. The concept of stability of vitiligo: a reappraisal. Indian J Dermatol. 2012;57(2):83-89. doi:10.4103/ 0019-5154.94271

13. Lee DY, Choi SC, Lee JH. Generalized vitiligo treated by combination therapy of epidermal graft and systemic corticosteroid. Clin Exp Dermatol. 2009;34(7):838. doi:10.1111/j.1365-2230.2009.03478.x

14. Lee DY, Lee KJ, Choi SC, Lee JH. Segmental vitiligo treated by the combination of epidermal grafting and systemic corticosteroids. Dermatol Surg. 2010;36(4):575-576. doi:10.1111/j.15244725.2010.01506.x

15. Banerjee K, Barbhuiya JN, Ghosh AP, Dey SK, Karmakar PR. The efficacy of low-dose oral corticosteroids in the treatment of vitiligo patient. Indian J Dermatol Venereol Leprol. 2003;69:135-137.

16. Wang AS, Armstrong EJ, Armstrong AW. Corticosteroids and wound healing: clinical considerations in the perioperative period. $\mathrm{Am}$ J Surg. 2013;206(3):410-417. doi:10.1016/j.amjsurg.2012.11.018

17. Forschner T, Buchholtz S, Stockfleth E. Current state of vitiligo therapy--evidence-based analysis of the literature. $J$ Dtsch Dermatol Ges. 2007;5(6):467-475. doi:10.1111/j.1610-0387.2007.06280.x

18. Rajagopal R, Murthy S, Kar K, Vijendran P. Autologous miniature punch grafting in stable vitiligo. Indian $J$ Dermatol. 2005;50:196-199.

19. Chandrashekar B, Madura C, Varsha D. Autologous mini punch grafting: an experience of using motorized power punch in 10 patients. J Cutan Aesthet Surg. 2014;7(1):42-45. doi:10.4103/09742077.129977

20. Van-geel N, Vandendriessche D, Vandersichel E, et al. Reference method for digital surface measurement of target lesions in vitiligo: a comparative analysis. Br J Dermatol. 2019;180(5):1198-1205. doi:10.1111/bjd.17190

21. Harris JE, Rashighi M, Nguyen N, et al. Rapid skin repigmentation on oral ruxolitinib in a patient with coexistent vitiligo and alopecia areata (AA). $J$ Am Acad Dermatol. 2016;74:(2):370-371. doi:10.1016/j.jaad.2015.09.073

22. Eleftheriadou V, Thomas KS, Whitton ME, Batchelor JM, Ravenscroft JC. Which outcomes should we measure in vitiligo? Results of a systematic review and a survey among patients and clinicians on outcomes in vitiligo trials. $B r J$ Dermatol. 2012;167 (4):804-814. doi:10.1111/j.1365-2133.2012.11056.x

23. Zawar VP, Karad GM. Needling in unresponsive stable vitiligo. $J$ Am Acad Dermatol. 2016;75(5):e199-e200. doi:10.1016/j.jaad.20 16.05.033

24. Frączek A, Owczarczyk-Saczonek A, Placek W. The role of TRM cells in the pathogenesis of vitiligo-a review of the current state-ofthe-art. Int J Mol Sci. 2020;21(10):3552. doi:10.3390/ijms21103552

25. Rajaram M, Selvaraj U, Alagarsamy S. Punch skin graft in stable vitiligo: donor and recipient site changes-A retrospective Study. Int J Sci Study. 2017;5(5):232-238. 
26. Das SS, Pasricha JS. Punch grafting as a treatment for residual lesions of vitiligo. Indian J Dermatol Venereol Leprol. 1992;58 (5):315.

27. Malakar S, Dhar S. Treatment of stable and recalcitrant vitiligo by autologous miniature punch grafting: a prospective study of 1000 patients. Dermatology. 1999;198(2):133-139. doi:10.1159/000018089

28. Boersma BR, Westerhof W, Bos JD. Repigmentation in vitiligo vulgaris by autologous minigrafting: results in nineteen patients. J Am Acad Dermatol. 1995;33(6):990-995. doi:10.1016/01909622(95)90292-9
29. Ebrahim HM, Albalate W. Efficacy of microneedling combined with tacrolimus versus either one alone for vitiligo treatment. $J$ Cosmet Dermatol. 2020;19(4):855-862. doi:10.1111/jocd.13304

30. Gupta S, Kumar B. Epidermal grafting in vitiligo: influence of age, site of lesion, and type of disease on outcome. J Am Acad Dermatol. 2003;49(1):99-104. doi:10.1067/mjd.2003.415

\section{Publish your work in this journal}

Clinical, Cosmetic and Investigational Dermatology is an international, peer-reviewed, open access, online journal that focuses on the latest clinical and experimental research in all aspects of skin disease and cosmetic interventions. This journal is indexed on CAS.
The manuscript management system is completely online and includes a very quick and fair peer-review system, which is all easy to use. Visit http://www.dovepress.com/testimonials.php to read real quotes from published authors. 